\title{
Rhinosinusal Melanomas
}

\section{Carlos S Ruggeri", Eduardo Figueroa, Agustina Lorea, Gabriel Rondón Gonzalez and Ana Carolina Riveros}

Rhinosinusology Section, Department of Otorhinolaryngology, Hospital Italiano of Buenos Aires, Argentina

*Corresponding author: Carlos S Ruggeri, Rhinosinusology Section, Department of Otorhinolaryngology, Hospital Italiano of Buenos Aires, Perón 4190, Ciudad Autónoma de Buenos Aires, Argentina

\begin{abstract}
Purpose: Melanomas are malignant tumors that have a poor prognosis and surgical treatment is the best option when they are resectable.

In this study we determined the incidence and survival what we achieved in our population of patients treated for melanomas of the nasal cavity and paranasal sinuses.
\end{abstract}

Material and methods: The medical records of all patients diagnosed with rhinosinusal malignant tumors at the hospital Italiano of Buenos Aires from March 1999 to December 2020 were reviewed.

Patients with melanomas were selected $y$ and we determine its incidence within malignant rhinosinusal tumors. Clinical characteristics, location, type of treatment indicated, and results were analyzed.

Results: Eight patients had melanomas, five located in the paranasal sinuses, two in the nasal cavity and one in nasal cavity and maxillary sinus.

The incidence of melanomas in our population of patients with rhinosinusal malignant tumors was $11.11 \%$.

Three patients were treated with surgery and radiotherapy and three with surgery only.

Two were treated with palliative criteria.

In the six patients treated with curative intent, the local control and specific survival were $83.33 \%$, and $66.66 \%$ respectively.

The average follow up time was 30.99 months.

Conclusions: The incidence of melanomas in our population of patients with rhinosinusal malignancies was $11.11 \%$.

In patients treated with curative intention, the local control rate was $83.33 \%$, and disease-free survival was $66.66 \%$.

The more frequently failure pattern was the appearance of distant metastases.
Endonasal surgery with endoscopes alone or associated with an external approach facilitated oncological resection.

\section{Keywords}

Melanoma, Paranasal sinuses, Nasal cavity, Endoscopic surgery

\section{Introduction}

Mucous melanomas of the nasal cavity and paranasal sinuses are malignant tumors that have a poor prognosis.

It is common that despite obtaining local control after treatment distant metastases appear.

Surgery is the first line of treatment if the tumor is resectable, and adjuvant radiation therapy and or chemotherapy are controversial.

\section{Objectives}

To determine the incidence of mucous melanomas of the nasal cavity and paranasal sinuses and analyze the results of treatment in our patient population.

\section{Material and Methods}

The medical records of all patients diagnosed with rhinosinusal malignant tumors at the hospital Italiano of Buenos Aires from March 1999 to December 2020 were reviewed.

Patients with melanomas were selected and clinical characteristics (presenting signs and symptoms), location, type of treatment indicated, and results were analyzed.

All patients were evaluated by otorhinolaryngological 
examination, nasal endoscopy, Computed Tomography $(\mathrm{CT})$, and magnetic resonance imaging with contrast (MR).

The study was completed with tomography of the brain, chest, abdomen and pelvis and in others with Positron Emission Tomography (PET-CT).

In all the histopathological study and immunostaining confirmed melanoma.

The treatments indicated were surgery, and surgery plus adjuvant radiotherapy.

Palliative treatment was with endonasal surgery with endoscopes and radiotherapy.

Surgeries were performed by endonasal endoscopic approach (resection of melanomas of the nasal cavity and sphenoid sinus), and through a combined approach: external and endonasal assisted with endoscopes. In all of them, intraoperative control of the margins was performed by frozen biopsies.

In one of the patients treated by a combined approach, a partial maxillectomy, ethmoidectomy, frontal drainage type Draf III, and exenteration of the orbit were performed, and in another a medial maxillectomy, ethmoidectomy, sphenoidotomy, lamina papyracea, periorbit and ipsilateral cribriform plate and dura mater resection were performed. The skull base was reconstructed with a multilayer technique, with a cartilage graft that was placed between the dura and the bone and a septal mucoperiosteal free graft that was placed on the bone ("over").

The facial defect was repaired with local flaps (middle frontal and Mustarde).

In another, anendonasal endoscopic medial maxillectomy with resection of the tear duct and posterior wall of the maxillary was performed with drilling of the sinus floor bone, and anterior external wall resection of maxillary sinus.

Controls were made by nasal endoscopy, magnetic resonance imaging and $\mathrm{PET}-\mathrm{CT}$.
They were done in conjunction with the clinical oncologist.

\section{Results}

Seventy two patients were diagnosed with malignant neoplasms of the nasal cavity and paranasal sinuses.

Eight of them had melanomas, five located in the paranasal sinuses, two in the nasal cavity and one in nasal cavity and maxillary sinus.

The incidence of melanomas in our population of patients with rhinosinusal malignant tumors was $11.11 \%$.

Six were men and 2 women, the youngest was 50 -years-old and the oldest was 87 , the average age was 70 years.

In five the tumor was located in the paranasal sinuses (ethmoid 3/5, frontal 1/5, and sphenoid 1/5), in 2 in the nasal cavity (inferior turbinate and anterior floor) and in one in nasal cavity (inferior turbinate) and maxillary sinus.

One had an invasion of the eyeball and another invasion of the lamina papyracea.

All the patients had unilateral nasal obstruction, two also had epistaxis and another had proptosis.

Only one (melanoma of the floor of the nostril) had a history of two previous surgeries performed at another hospital.

In 7 patients, a biopsy was done prior to treatment, and in one that had a tumor located in the lateral wall of the nasal cavity, an excisional biopsy and an intraoperative study of the surgical specimen were performed. The report was melanoma, so the resection margins were widened and controlled by intraoperative biopsies at the same surgical time.

Six were treated with surgery (endonasal surgery with endoscopes $(3 / 6)$, and endonasal surgery with endoscopes combined with an external approach (3/6) (Figure 1, Figure 2, Figure 3, Figure 4 and Figure 5).

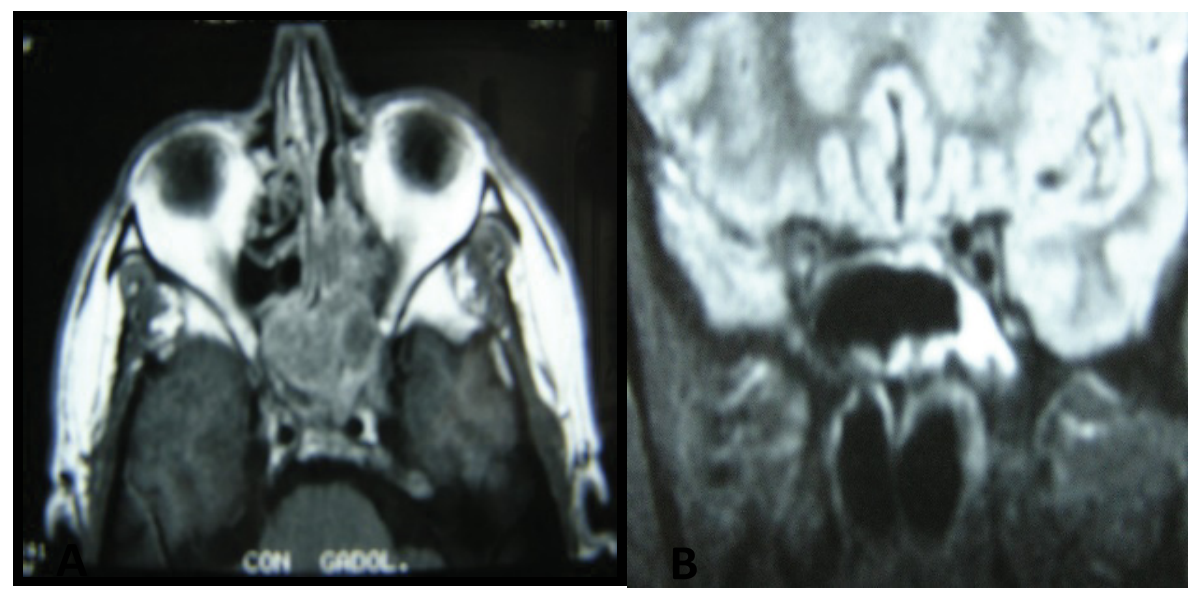

Figure 1: Sphenoid melanomatreated with endoscopic surgery + radioteraphy (A) Preoperative CT (B) Postoperative CT. 


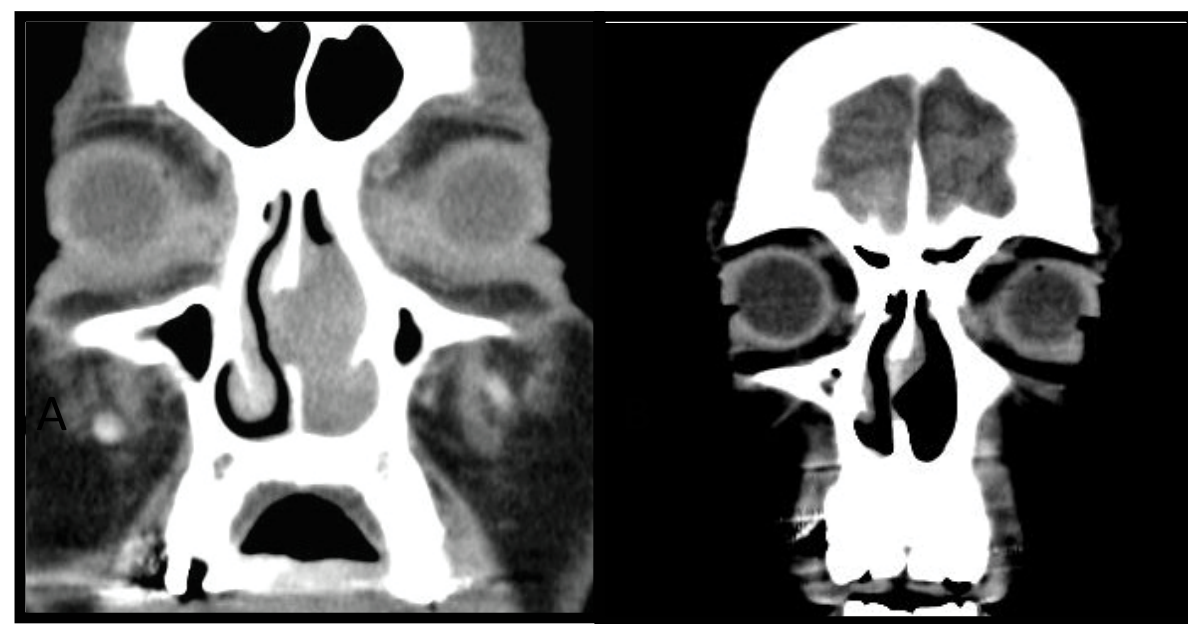

Figure 2: Nasal cavity melanoma (A) Preoperative CT; (B) Postoperative CT.

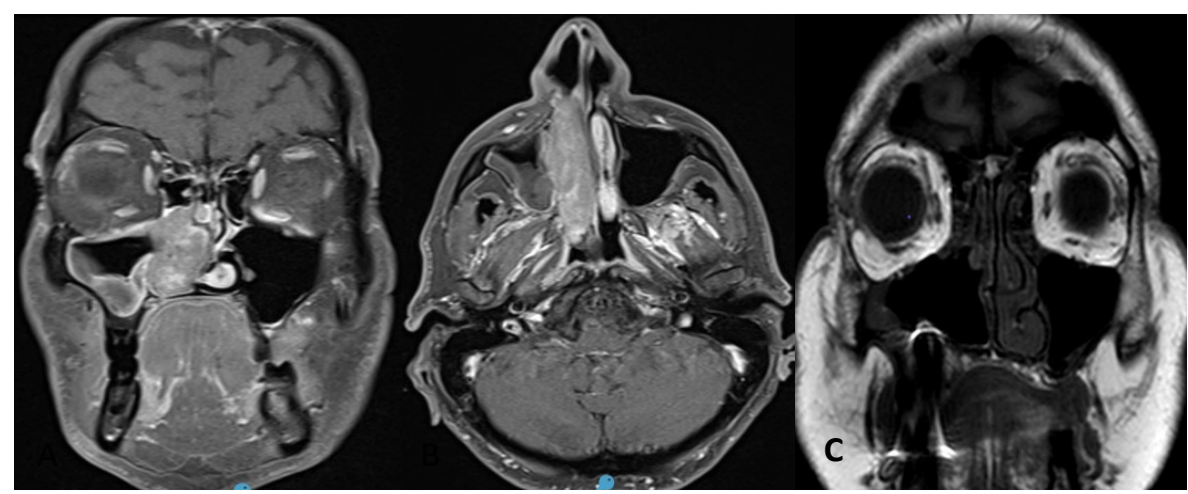

Figure 3: Inferior turbinate and maxillary sinus melanoma.

Medial maxillectomy, tear duct and anterior and posterior wall resection with drilling of the sinus floor bone. (A,B) CT preoperative; (C) Postoperative CT

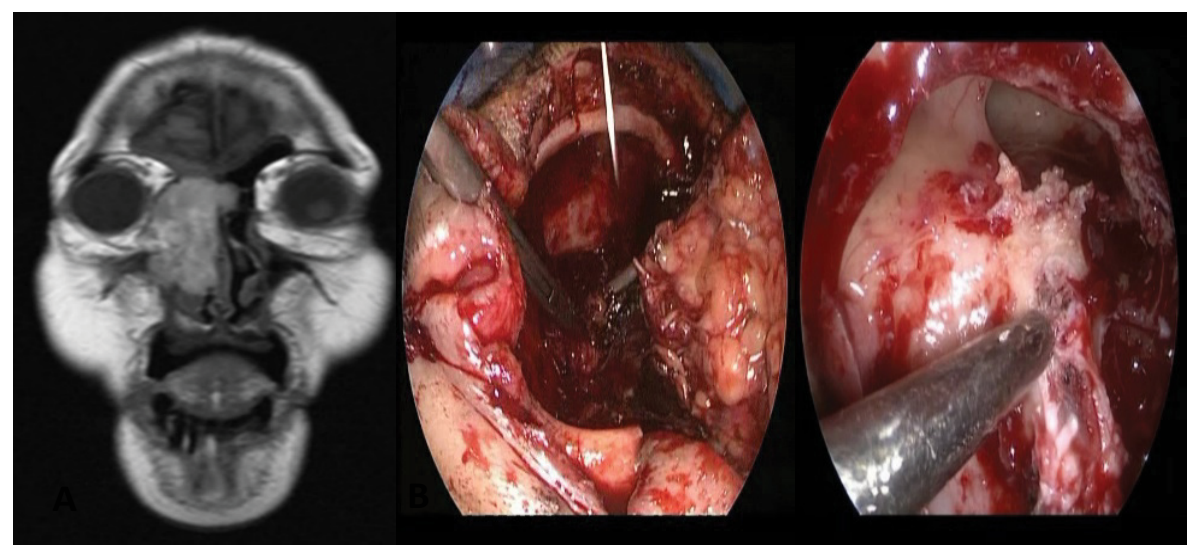

Figure 4: Ethmoid melanoma with eyeball invasion (A) MR; (B) Partial maxillectomy with orbital exenteration (C) Endoscopic vision Lothrop modified.

In the 3 patients with melanomas of the paranasal sinuses, adjuvant treatment with radiotherapy was also indicated.

An 87-year-old patient with poor general condition refused treatment and underwent palliative surgery by endonasal approach to unblock the nostrils.

The patient with melanoma located in the frontal sinus had a disseminated disease (cervical and pulmonary metastases), for which palliative treatment with radiotherapy was used.

In the six patients treated with curative intent, the local control rate was $83.33 \%$, and disease-free survival was $66.66 \%$.

One of them had a single brain metastasis two 


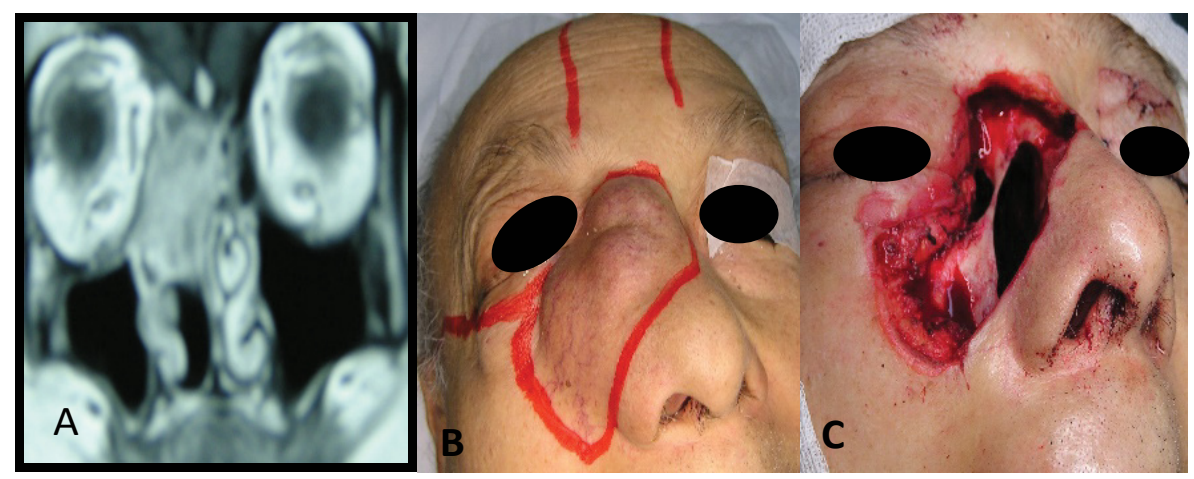

Figure 5: Ethmoid melanoma with facial skin and skull base invasion (A) MR; (B) Skin invasion; (C) Combined external and endonasal approach with endoscopes (facial skin resection, ethmoidectomy, papyracea and periorbit and skull base resection).

Table 1: Patients treated with paranasal sinuses and nasal cavity melanomas.

\begin{tabular}{|l|l|l|l|l|l|l|}
\hline Sex/age & Location & Surgery & Radiotherapy & $\begin{array}{l}\text { Local } \\
\text { recurrence }\end{array}$ & $\begin{array}{l}\text { Distal } \\
\text { metastasis }\end{array}$ & Follow up \\
\hline Male/50 & Sphenoid & Es & $\mathrm{Si}$ & No & No & No \\
\hline Male/83 & Ethmoid & Es $+\mathrm{Ea}$ & $\mathrm{Si}$ & No & $\begin{array}{l}18 \text { months/no return to } \\
\text { control }\end{array}$ \\
\hline Male/73 & Nasal Cavity & Es & No & No & Si & $>5$ Years \\
\hline Female/87 & Ethmoid & Palliative Es & No & - & No & 2 years/died \\
\hline Male/68 & Frontal sinus & Biopsy & - & - & Si & 4 months/died \\
\hline Male/70 & Nasal cavity & Es & No & No & No & 24 months \\
\hline Female/64 & Ethmoid & Ea & Si & Si & Si & 12 months/died \\
\hline Male/65 & $\begin{array}{l}\text { Nasal cavity and } \\
\text { maxillary sinus }\end{array}$ & Es + Ams & No & No & No & 12 months \\
\hline
\end{tabular}

ES: Endoscopic Surgery, EA: External Approach, AMS: Anterior Maxillary Sinusotomy

years after nasal surgery, underwent surgery, and lived disease-free for another 2 years. Later he suffered lung and bone metastases. Vemurafenib was prescribed.

He lived 4 more years and died of a stroke. He never had local recurrences.

Another patient had a local persistence of the disease and multiple systemic metastases 10 months after surgery and postoperative radiotherapy.

Follow-up in patients treated for curative purposes was greater than 5 years in two, 18 months in one, 12 months in two and in another 24 months.

Of the patients who received palliative treatment, one did not return to control and the other died after 2 years (Table 1 ).

\section{Discussion}

Melanoma is an aggressive malignant tumor that arises from melanocytes that originate from the neural crest.

Melanocytes are found in the mucosa, secretory glands, nasal stroma, and in the supporting cells of the olfactory epithelium.

Primary mucosal melanoma is a rare aggressive malignant tumor, accounting for $4 \%$ to $8 \%$ of rhinosinusal malignant neoplasms and $0.7 \%$ of melanomas. Fifty five percent originate in the head and neck, and two thirds of these are located in the paranasal sinuses and nasal cavity and a quarter in the oral cavity $[1,2]$.

The most frequent age of diagnosis is between 65 and 70 years, later than in cutaneous melanoma and the incidence is similar between women and men.

In our series of patients, the incidence of mucous melanoma among diagnosed rhinosinusal malignant tumors was $11.11 \%$, slightly higher than those mentioned in the literature.

Clinically, like other tumors, they can simulate an inflammatory process and delay diagnosis.

The most common signs and symptoms of consultation are epistaxis and unilateral nasal obstruction.

On endoscopic examination, they may appear pigmented, although $10 \%$ percent may be amelanotic. All patients in our study had tumors with black pigmentation.

They are most frequently located in the nasal cavity, especially on the lateral wall. The nasal septum, maxillary sinus and ethmoid follow in order of frequency. 
In our study, melanomas originating in the paranasal sinuses predominated $(5 / 8)$, and the most common location was the ethmoid. In two patients the tumors originated in the sphenoid and the frontal sinus, which are exceptional locations.

Cutaneous melanomas are staged according to their thickness, thickness and ulceration and according to the level of invasion of the dermis. These staging systems are based on the study of large series of patients and are related to prognosis.

Rhinosinusal mucosal melanomas are very infrequent, there are no limits as in the dermis that allows the depth of invasion to be evaluated and this does not correlate with the prognosis.

A relationship between the TNM classification used for malignant tumors of the paranasal sinuses and nasal cavity and the prognosis for melanomas has not been described, except in one study [3].

Treatment of rhinosinusal mucosal melanomas is primarily surgical, and the goal is to obtain diseasefree margins. This is most often possible in nasal cavity tumors and challengingly in neoplasms located in the paranasal sinuses.

The use of an external approach (paralateronasal, sublabial, Weber Ferguson, craniofacial) or an endonasal approach with endoscopes depends on the location and extent of the melanoma.

Regardless of the approach, surgery must achieve a complete resection, with wide margins and free of tumor.

Endonasal surgery with endoscopes and endocameras allows a vision with a better angle and with magnification that makes it possible to perform more detailed resections, especially in this type of tumors that can present amelanotic satellite lesions, and submucosal extensions.

In a study on 58 patients with rhinosinusal melanomas, they found no differences in local control between patients operated on by endoscopic surgery or with an external approach [4].

Lombardi, et al. did not describe differences in survival in 58 patients with rhinosinusal melanomas operated by the endonasal route (with or without craniectomy) or with a cranioendoscopic resection [5].

In our study we used endoscopes in all patients treated with surgery, in three only and in the others combined with an external approach.

Melanoma is considered a radioresistant tumor, but it can respond to high doses of radiation. Radiation therapy is more often indicated as adjunctive treatment after surgery.

In a meta-analysis, they described that postoperative radiotherapy obtained a benefit in overall survival at 3 years; at 5 years the benefit was minimal when compared with surgery alone. No statistically significant advantage was obtained in specific disease-free survival and in local control [6].

In two other meta-analyses on mucous melanomas of the head and neck and upper aero-digestive tract, they concluded that adjuvant radiotherapy reduced the local recurrence rate but did not improve survival $[7,8]$.

When patients are controlled for more than 5 years after treatment, the local recurrence rate is low (20\%), but the appearance of distant metastases is greater than $80 \%$ [9].

This may explain why better local control is not associated with better survival.

Postoperative radiotherapy was indicated in three of the six patients in our study who were treated with curative intent. In two due to the melanoma extent (eyeball and facial skin and skull base invasion) and in another due to its location in the sphenoid sinus, where the free margins obtained after resection were reduced.

In melanomas of the nasal cavity, the resections had wide margins and no other treatment was indicated.

The prognosis for rhinosinusal melanomas is poor, worse than for cutaneous melanomas.

The 5-year survival of rhinosinusal melanomas is generally less than $25 \%$.

Some studies report survival rates between $8 \%$ and $48 \%$, with an overall mean survival of 12.5 to 19.3 months [10].

In our small series disease-free survival in patients treated with curative intent was $66.66 \%$ and local control $83.33 \%$, the mean follow-up time was 30.99 months.

We believe that the reduced number of patients treated and the shorter follow-up on some could explain the better disease-specific survival and local control rates that we obtained.

Systemic treatment of mucous melanoma is indicated in patients with unresectable or metastatic disease.

Mucous melanomas may have mutations in the C-KIT gene, its presence does not change the prognosis, but it selects a group of patients that can be treated with tyrosine kinase inhibitors.

In a phase II study they examined the efficacy of imatinib (a tyrosine kinase inhibitor) in the treatment of mucosal melanomas, and reported a significant response rate of $29 \%$ and a total disease control rate of $50 \%[11]$.

\section{Conclusions}

The incidence of melanomas in our population of patients with rhinosinusal malignancies was $11.11 \%$. 
In patients treated with intention to cure, the local control rate was $83.33 \%$, and disease-free survival was $66.66 \%$.

The more frequent failure pattern was the appearance of distant metastases.

Endonasal surgery with endoscopes alone or associated with an external approach facilitated oncological resection.

\section{Conflicts of Interest}

We have no conflicts of interest.

\section{References}

1. Gilain L, Houette A, Montalban A, Mon T, Saroul N (2014) Mucosal melanoma of the nasal cavity and paranasal sinuses. Eur Ann Otorhinolaryngol Head Neck Dis 131: 365-369.

2. Roth TN, Gengler C, Huber GF, Holzmann D (2010) Outcome of sinonasal melanoma: clinical experience and review of the literature. Head Neck 32: 1385-1392.

3. Moreno MA, Roberts DB, Kupferman ME, DeMonte F, ElNaggar AK, et al. (2010) Mucosal melanoma of the nose and paranasal sinuses, a contemporary experience from the M. D. Anderson Cancer Center. Cancer 116: 22152223.

4. Lundberg $M$, Haapaniemi A, Hagstrom J, Juteau $S$, Hernberg M (2019) Similar survival outcome after endoscopic and open approaches for sinonasal mucosal melanoma. Rhinology 57: 132-138.
5. Lombardi D, Bottazoli M, Turri-Zanoni M, Raffetti E, Villaret $A B$, et al. (2016) Sinonasal mucosal melanoma: a 12 year experience of 58 cases. Head and Neck 38: 1737-1745.

6. Hu R, Yang BB (2019) Surgery alone versus post-operative radiotherapy for sinonasal malignant melanoma: $A$ metaanalysis. J Laryngol Otol 132: 1051-1060.

7. Li W, YuY, Wang H, Yan A, Jiang X (2015) Evaluation of the prognostic impact of postoperative adjuvant radiotherapy on head and neck mucosal melanoma: A meta-analysis. BMC Cancer 15: 758.

8. Jarrom D, Paleri V, Kerawala C, Roques T, Bhide S, et al. (2017) Mucosal melanoma of the upper airwayz tract mucosal melanoma: a systemic review with meta-analysis of treatment. Head Neck 39: 819-825.

9. Christopherson K, Malyapa RS, Werning JW, Morris CG, Kirwan J, et al. (2015) Radiationtherapy for mucosal melanoma of the head and neck. Am J Clin Oncol 38: 8789.

10. Lund VJ, Howard DJ, Wei WT (2014) Mucosal malignant melanoma. In: Tumors of the nose, sinuses, and nasopharynx. Thieme, Stuttgart-New York 2014: 377-387.

11. Hodi FS, Corless CL, Giobbie-Hunder A, Fletcher JA, Zhu M, et al. (2013) Imatinib for melanomas harboring mutationally activated or amplified KIT arising on mucosal, acral, and chronically sun damaged skin. J Clin Oncol 31: 3182-3190. 